\section{Cahiers de Narratologie}

Analyse et théorie narratives

10.2 | 2001

La voix narrative

\title{
Interdit et clôture : récits enchâssés et discours contraint dans l'œuvre de Juana Manuela Gorriti
}

\section{Michèle Soriano}

\section{(2) OpenEdition}

1 Journals

Édition électronique

URL : http://journals.openedition.org/narratologie/10294

DOI : 10.4000/narratologie.10294

ISSN : 1765-307X

Éditeur

LIRCES

\section{Édition imprimée}

Date de publication : 1 janvier 2001

Pagination : 441-454

ISBN : 2914561032

ISSN : 0993-8516

\section{Référence électronique}

Michèle Soriano, «Interdit et clôture : récits enchâssés et discours contraint dans l'œuvre de Juana Manuela Gorriti », Cahiers de Narratologie [En ligne], 10.2 | 2001, mis en ligne le 01 janvier 2001, consulté le 11 juin 2020. URL : http://journals.openedition.org/narratologie/10294 ; DOI : https:// doi.org/10.4000/narratologie.10294 


\title{
INTERDIT ET CLÔTURE : RÉCITS ENCHÂSSÉS ET DISCOURS CONTRAINT DANS L'CEUVRE DE JUANA MANUELA GORRITI
}

\author{
Michèle SORIANO \\ Université de Montpellier III
}

...Y cuchicheaban entre ellas ahogando prolongados bostezos.

Chuchotements et murmures : clôture. L'insignifiance d'un dire se décline sous la forme de l'informe, susurrements étouffés, signifiants dont les traits miment la vacuité répétitive, et l'onomatopée, propos verbeux qui dans leur agressive surabondance seront devenus blabla, et dont l'illégitime circulation parviendrait au statut de fable, cancan, caquet, et autres rumeurs, bruissements d'humeurs. Un interdit définit ce bavardage oiseux, ce barbare babil. Cet énoncé fort bref nous livrerait ainsi la situation et la valeur d'une parole, l'espace qui lui est réservé - « entre ellas »-, et la double limite qu'elle transgresse lorsqu'elle advient : celle du corps, qui doit demeurer clos - « ahogando prolongados bostezos »-, celle du verbe, auquel elle ne saurait prétendre. Néanmoins, dans l'insistance du chuchotis chuintant, ou l'excessive durée de cette béance contenue, comment ne pas entendre une impatience certaine, une informulable, mais tenace, résistance à l'oppression qui la condamne au vide.

Babil: "Les jeunes filles acquièrent vite un petit babil agréable » (Rousseau).

Chuchotements : "De longs chuchotements de jeunes filles, des rires étouffés » (Lamartine)

Papoter: "Les belles dames de l'Opéra papotaient dans leurs loges » (Duhamel)

Caquetant : "Jeunes filles [...] caquetantes et jacassantes » (Roger Ikor) 
Ces quelques exemples issus du Petit Robert nous engagent à interroger la représentation consacrée d'une certaine parole, toujours ouie à distance, et connue par ouïdire, inter-dite ou inouïe. Deux essais assez récents convergent sur un point qu'il nous importe de mettre en valeur, dans les connaissances nouvelles que créent les fables de cette figure un peu marginale de la littérature argentine du siècle dernier, Juana Manuela Gorriti, par la mise en scène - mise à distance -, de différents discours, récits que nous analyserons à titre d'exemple d'une stratégie narrative qu'il est possible de repérer ailleurs ${ }^{1}$; il est en effet curieux de constater l'identité presque parfaite de ces titres de sous-parties: "Etre comme on est perçue " (M. Le Doeuff, Le sexe du savoir, 1998,165 ) et «L'être féminin comme être-perçu » (P. Bourdieu, La domination masculine, 1998, 70). La première propose une généalogie documentée du champ scientifique et explique :

L'histoire de la médecine ancienne montre la mise en place de cases vides, "le théorique", "le pragmatique " ou «le pratique ", définies bien avant qu'un contenu vienne les remplir. On n'est pas encore dans le savoir mais on définit déjà les catégories du savoir ; une organisation du rapport cognitif précède toute connaissance et la femme, objet d'un prétendu savoir spécial détenu par les hommes, assure à ceux-ci leur fonction théoricienne et leur statut de sujet connaissant, alors qu'ils ne connaissent (encore) rien. (161)

Le second démontre que :

Tout, dans la genèse de l'habitus féminin et dans les conditions sociales de son actualisation, concourt à faire de l'expérience féminine du corps la limite de l'expérience universelle du corps-pour-autrui, sans cesse exposé à l'objectivation opérée par le regard et le discours des autres. (70)

Les fables de Gorriti, qui s'inscrivent dans un cadre institutionnel, mais dans une position excentrée, qui semblent convoquées par une loi qu'elles ne citent que pour mieux la

${ }^{1}$ Dans les récits fantastiques de Silvina Ocampo, par exemple, ou encore dans les contes de Rosario Castellanos. 
détourner, feignent également de reproduire une situation topique : un regard et un discours masculin sur un objet féminin ; cependant, dans l'écart qu'institue leur mise en scène, le texte produit l'espace nécessaire à l'articulation d'un discours contradictoire, renversant les pôles des processus d'objectivation. Nous proposerons d'examiner brièvement le fonctionnement de la voix narrative dans quelques récits afin de mettre en valeur la production d'un lieu où d'autres paroles pourraient s'entendre, et être reconnues.

\section{Confession}

Les deux textes intitulés «Coincidencias ", et le conte "Quien escucha su mal oye », considérés par la critique comme précurseurs du Fantastique hispano-américain (O. Hann, P. Verdevoye), s'organisent en une série de récits métadiégétiques, juxtaposés et / ou enchâssés. L'instance narrative du récit premier est d'abord anonyme et se représente souvent comme l'un des membres d'un groupe, adoptant la première personne du pluriel, elle devient ensuite auditrice des récits seconds qu'elle rapporte, mais elle se dédouble dans la dernière section du récit, et apparaît alors en tant que narrateur intradiégétique.

Dans la série «Coincidencias » issue de Panoramas de la vida (tome II, 1876) quatre récits sont juxtaposés et portent chacun un sous-titre : "El emparedado », "Fantasma de un rencor », "Una visita infernal » et « Yerbas y alfileres ». Le premier est précédé d'un préambule de l'instance narratrice qui décrit une situation topique : «Eramos diez. Habíanos reunido la casualidad, y nos retenía en un salón, en torno a una estufa improvisada, el más fuerte aguacero del pasado invierno. " La description de la situation - dont est issue la phrase citée plus haut - oppose ensuite à la patience du groupe l'impatience de quatre jeunes filles, qu'un vieux vicaire invite alors à chanter, leur tendant un cahier. «Coincidencias » s'écrient les jeunes filles, car le cahier est vide. Et ce mot va engendrer un dialogue qui ouvrira la série de récits métadiégétiques, associés par cette thématique commune, et dont la fonction par rapport à la diégèse serait de 
distraire ces dix personnes ainsi contraintes à l'immobilité, et à l'attente.

- «Coincidencias»! Eso más bien que de cantos, tiene sabor de relato - dijo una señora mayor.

- Y quien dijo relatos - añadió otra - quiso decir plática de viejos.

- Y quien dijo plática de viejos, quiso aludir a mis noventa inviernos-repuso con enfado cómico el vicario.

- Y para castigar la culpable susceptibilidad de ese Ministro del Señor - replicó la matrona - simulando el énfasis de un fiscal - pido que se le aplique la ley al pie de la letra, y se le condene al relato de una coincidencia. (128-129)

Dès ce dialogue initial il est possible d'observer que, sous un mode parodique, une fonction seconde est attribuée à l'acte narratif : il permet d'expier une faute. Or, si les récits fantastiques qui suivent jouent tous, en effet, sur des coïncidences troublantes, suggérant l'existence de phénomènes surnaturels - fantômes, pouvoirs occultes, malédiction, maléfices, et visite mystérieuse du Diable -, ils ont en outre en commun la mise en évidence d'une transgression: directement ou indirectement ils révèlent une faute. Dans «Quien escucha su mal oye "», publié dans Sueños y realidades (1864), le récit métadiégétique reçoit de façon tout à fait explicite cette même fonction, le conte commence ainsi :

Cuando hemos caído en una falta - me dijo un día cierto amigo -, si la reparación es imposible, réstanos, al menos, el medio de expiarla por una confesión explícita y franca. ¿Quiere usted ser mi confesor, amiga mía ? (104)

Et l'un des deux récits réunis sous ce même titre de «Coincidencias », dans le recueil Misceláneas fonctionne, de même, comme la confession d'un crime commis par le narrateur intradiégétique. Ainsi, tous ces textes intègrent des récits seconds qui tous se réfèrent, d'une façon ou d'une autre, à une faute originelle, et tous renvoient, sur le mode parodique, à cette pratique rituelle qui fait de l'acte narratif un discours contraint participant du contrôle des actes et des pensées du sujet, car dans cette mise en récit de soi il se soumet à des règles strictes, à l'autorité institutionnelle, et à la volonté de savoir de son directeur de conscience. 
Or la confession, en tant que pratique non seulement rituelle mais aussi sociale, dans la société hispano-américaine du XIXème siècle, qui convoque une parole contrainte, autorisée parce que médiatisée et secrète, à la fois imposée et soumise à la plus étroite des clôtures, génère et fixe les modalités de l'une des pratiques discursives les plus représentatives des conditions d'énonciation que la culture catholique androcentrique réserve aux femmes, et ce d'autant plus qu'elle renvoie à la faute celles qu'elle confond avec l'origine même de celle-ci. La déconstruction de cette pratique dans les récits de Gorriti n'est donc ni aléatoire ni anodine, un double enjeu socio-historique peut se lire dans les discours qui l'organisent et qu'il conviendra de tenter d'identifier.

Par ailleurs, à l'origine du conte «Quien escucha su mal oye », dans cette phrase du narrateur second qui inaugure le texte, s'énonce la notion de chute, et c'est bien sur cette "faute originelle" que s'articule la totalité du conte, car nous retrouvons dans les quatre récits enchâssés qui le composent la transgression de la loi sous la forme de l'acquisition d'une connaissance interdite. La chute d'Adam et Eve est repérable en tant que texte culturel dans ce conte de Gorriti, et apparaît de façon récurrente dans la littérature produite à partir d'une position féminine. Comme si l'écriture supposait alors la transgression d'un interdit, et le renouvellement de cette transgression originelle, en outrepassant les limites assignées à une partie de la population, en raison de son identité sexuelle; identité représentée dans l'imaginaire androcentrique, - et en deçà, réalisée dans les schèmes qu'un monde sexuellement hiérarchisé imprime dans l'ordre des corps (Bourdieu, 1998, 61-63)-, comme naturellement exclue du savoir, et donc de son exercice, à travers l'écriture. La prégnance, dans nos sociétés, de ce mythe originel étant due, entre autres pratiques dévalorisantes, au relais incessant que constituait (et constitue encore en partie) une exclusion de fait des institutions accordant quelque capital symbolique et l'autorisation d'en user (M. Le Doeuff, 1998, 343-345; P. Bourdieu, 1998, 102-103). Ainsi, la présence de cette fable originelle, de ses présupposés et de ses conséquences, estelle en quelque sorte surdéterminée, de même que leur déconstruction, par le travail d'une écriture qui, pour être 
produit à partir d'une position tenue pour impossible, questionne nécessairement l'ordre social que ce mythe articule et dont il fonctionne comme l'un des emblèmes ${ }^{2}$.

Les quatre récits enchâssés de "Quien escucha su mal oye ", et non pas uniquement le récit cadre, peuvent être lus comme une déconstruction de la pratique de la confession, explicitement convoquée dans le dialogue initial par celui qui deviendra ensuite le narrateur second, mais également représentée dans les autres niveaux narratifs qui mettent en scène des aveux. Cette déconstruction problématise le titre du conte, en forme de proverbe, et les notions de transgression et d'expiation qui fondent autant la pratique de la confession que l'exclusion de la parole des femmes prononcée par Saint Paul et perpétuée par les pratiques sociales et les institutions qui définissent le cadre autorisé et autoritaire qui voue la parole féminine au silence ou à l'aveu, en lui interdisant toute énonciation publique :

Comme cela se fait dans toutes les églises des saints que les femmes se taisent dans les assemblées : elles n'ont pas la permission de parler; elles doivent rester soumises, comme dit aussi la loi. Si elles désirent s'instruire sur quelque détail, qu'elles interrogent leur mari à la maison. Il n'est pas convenable qu'une femme parle dans les assemblées. La parole de Dieu a-t-elle chez vous son point de départ ? (1, Corinthiens, 14, 34-35)

Pendant l'instruction, la femme doit garder le silence, en toute soumission. Je ne permets pas à la femme d'enseigner ni de dominer l'homme. Qu'elle se tienne donc en silence. C'est Adam, en effet, qui fut formé le premier. Eve ensuite. Et ce n'est pas Adam qui fut séduit, mais c'est la femme qui, séduite, tomba dans la transgression. Cependant elle sera sauvée par sa maternité à condition de persévérer dans la foi, l'amour et la sainteté, avec modestie. (1 Timothée, 2, 11-15)

Or, dans «Quien escucha su mal oye » la chute est associée à un agent masculin trop curieux, et une femme se

2 On se reportera aux réflexions à propos de la déconstruction de cette fable que Michèle Le Dœuff élabore dans Le sexe du savoir, commentant un essai de Gabrielle Suchon, puis un essai de Jeanne Hersch, p. 71-93 et 121-124. 
trouve dans la position de juge et confesseur, il en va de même dans les deux séries de récits intitulées "Coincidencias" : le narrateur second est d'abord un personnage masculin qui est contraint d'expier sa faute en produisant un récit soumis à l'écoute d'une interlocutrice. Cette double substitution contribue à produire un questionnement systématique des catégories légitimes et de la différenciation qu'elles structurent. D'autre part, nous observons que les quatre récits enchâssés de "Quien escucha su mal oye » reproduisent un même schéma : l'action consiste à trahir un secret, lui-même portant sur la violation d'un autre secret, locuteurs et interlocuteurs sont aussi coupables que les protagonistes qu'ils évoquent et qui fonctionnent à leur tour comme locuteurs et interlocuteurs à un autre niveau du récit, tous pénètrent ou trahissent un secret qui devait demeurer interdit. Ces substitutions suggèrent la réversibilité de leur fonction, et l'identité de leur position. L'indistinction qui résulte de la spécularité narrative questionne les critères de différenciation qui distinguent les divers personnages, critères par ailleurs explicites et fort présents, qui procèdent du monde référentiel et de ses systèmes axiologiques; la mise en abyme tend à neutraliser les catégories que ces systèmes définissent : homme/ femme, maître/ esclave, actif/ passif, divin/ diabolique, passé/ présent, péché/ innocence, savoir/ ignorance ${ }^{3}$. Les quatre récits disposés en série du conte "Coincidencia» (Panorama de la vida) proposent tout d'abord une sorte de symétrie spéculaire qui fait se succéder dans la position de narrateur intradiégétique deux personnages masculins, deux "ministres de Dieu", puis deux personnages féminins qui reçoivent un statut inverse, et opposent à la parole édifiante et orthodoxe une parole ludique et hérétique. En effet, ainsi termine le second récit et s'annonce le troisième, intitulé «Una visita infernal»:

Las jóvenes lloraban escuchando el triste relato del canónigo.

3 J'ai analysé en détail ce texte dans «El saber de la ficción en Quien escucha su mal oye : estudio sociocrítico de los inicios del género fantástico ", in Juanamauela, mucho papel, Amelia Royo compiladora, Ed. del Robledal, Salta, 1999, p. 241-284. 
- Válgame Dios - exclamó una señora - y qué fuerte olor de sacristía han esparcido en nuestro ánimo estas historias de clérigos. Será preciso para neutralizar el incienso, saturarlo con esencia de rosas. Y pués que de coincidencias se trata allá va una de tantas.

Hable el siglo - repuso el vicario con un guiño picaresco.

Le quatrième récit est un cas particulier, car il fait intervenir comme narrateur intradiégétique l'instance narratrice initiale, qui cède ensuite la parole à un narrateur ; il s'agit cette fois d'un personnage masculin désigné comme "el esclerecido profesor Passaman », personnage qui à son tour, grâce à l'hypnose, suscite le récit d'un personnage féminin, convoqué afin de disqualifier, au nom de la science, certaines croyances jugées superstitieuses. Nous retrouvons alors les phénomènes d'enchâssement qui caractérisent "Quien escucha su mal oye ». Afin d'analyser les effets qu'ils produisent il convient d'expliciter le contexte axiologique de référence et d'interroger, en deçà des récits métadiégétiques, les discours qui structurent les énoncés.

\section{Discours et savoirs}

Michèle Le Dœuff propose de désigner par le terme de nomophatique " un code déterminant qui a le droit de parler, à qui, où, sur quel sujets, pour dire quoi et sur quel ton » $(1998,116)$, et décrit l'héritage de la nomophatique paulinienne évoquée plus haut. Dans les contes de Juana Manuela Gorriti cet héritage est mis en scène à travers le jeu des récits métadiégétiques. Ceux qui détiennent le droit à la parole sont en premier lieu les représentants d'un discours autorisé, masculins, et jouissant du capital symbolique que leur confère l'institution qu'ils représentent : prêtres, professeurs, docteurs. Les autres déterminations ne les concernent pas, ce droit peut s'exercer sur tout et en toutes circonstances; en revanche, elles s'appliquent à celles que leur grand âge autorise à émettre un discours, mais en privé, et sous réserve de limiter celui-ci à l'édification des plus jeunes, leur inculquant ainsi la soumission à l'ordre patriarcal qui ne saurait autrement créditer leurs propos. C'est ainsi que l'instance narratrice 
cède volontiers la parole à des narrateurs masculins, et la dénégation que suppose cet usage du récit métadiégétique se manifeste également dans la position asexuée qu'elle adopte : "Gracias a Dios, hace tiempo, que yo digo como madama Geofroid - quand j'étais femme. » («Escenas de Lima », 175), position que justifie - ou que lui impose - sa maturité. Les lieux sont domestiques et clos, les femmes y parlent d'amour ou de superstitions. Mais ce cadre minutieusement respecté est aussi représenté en tant que coercition, violence symbolique qui s'exerce, et dont les conséquences destructrices sont manifestes.

Revenons sur la situation initiale du conte « Coincidencias » (Panorama de la vida) :

El piano estaba, en verdad, abierto, y el pupitre sostenía una linda partitura y valses a discreción ; pero hallábanse entre nosotros dos hombres de iglesia; y su presencia intimidaba a las chicas, y las impedía entregarse a los compases de Straus y las melodías de Verdi. Ni aún osaban apelar al supremo recurso de los aburridos : pasearse cogidas del brazo, a lo largo del salón; y cuchicheaban entre ellas ahogando bostezos. (128)

$\mathrm{Au}$ piano ouvert s'oppose la clôture répressive qu'impose l'institution religieuse, qui réduit l'espace des jeunes filles au point de leur interdire tout mouvement, et prescrit un tel hermétisme à leur corps qu'elles ne sauraient ouvrir leur bouche, quand cela ne serait que pour bailler. Seul le souffle assourdi des chuchotis témoigne encore de leur présence. Cette clôture qu'organise l'Église est encore mise en scène dans le premier récit métadiégétique et dans l'effet qu'il produit. Le vicaire raconte comment, coupable de paresse, il doit s'enfermer dans une cellule, la nuit précédant un sermon solennel, afin le rédiger sans plus tarder. Alors qu'il sombre dans le sommeil il reçoit la visite d'un jésuite, qui lui indique l'origine de la citation qu'il cherchait : il la trouvera dans le chapitre 8 des Confessions de Saint Augustin, ensuite, l'étrange visiteur disparaît. Le lendemain, lors de travaux de réaménagement, le cadavre d'un jésuite est découvert, emmuré dans la cloison de la cellule que le vicaire occupait, à l'endroit de la disparition du visiteur qui avait déclaré «Yo duermo aquí ». La situation du jésuite n'est qu'un redouble- 
ment de celle du vicaire, condamné à l'enfermement, mais la violence qu'elle suppose est exhibée dans ce redoublement, car cet état condamne également son âme. Le commentaire de l'instance narratrice lève toute équivoque quand aux effets de ce récit, et donc quant à son sens et à la fonction répressive qu'il assume :

Las jóvenes, aunque se preciaban de espíritus fuertes, estrecharon sus sillas mirando con terror las ondulaciones que el viento imprimía a la cortinas del salón. (130)

La terreur que suscite cette image fonctionne comme une menace que l'orthodoxie réserve aux éventuels esprits indépendants, les fenêtres ouvertes de leur rébellion sont gardées par des fantômes et autres méchants loups.

Dans le deuxième récit métadiégétique un autre prêtre raconte la confession d'une jeune fille à l'agonie, « un ángel de candor, bondad y resignación " qui cependant, mais en rêve, maudit son frère, responsable du suicide de son fiancé. Pour des raisons politiques il a interdit l'union des deux jeunes gens. Le prêtre lui impose de pardonner et l'oblige à revoir son frère. Dans son délire son refus éclate : "Asesino de Enrique, aléjate, huye, o te doy mi maldición ", puis elle crie : « socorro para Eduardo cuyo caballo espantado por mi sudario acaba de arrojarlo a tierra donde yace sin sentido. " Le frère, qui a effectivement fait une chute, est secouru; il arrive, la sœur reçoit les sacrements dans ses bras et meurt en paix. Contre la légitimité de l'ordre masculin, seules des paroles délirantes et diaboliques peuvent être proférées. l'église fait le nécessaire pour contrôler jusqu'à la pertinence et l'opportunité de l'ultime soupir. Évidemment, les jeunes filles qui écoutent sont saisies, le désespoir est venu seconder la terreur.

"Una visita infernal » est le récit d'une dame qui rapporte la visite du diable reçue par sa sœur la nuit de ses noces. Lorsque celle-ci raconte son aventure à son mari il se moque d'elle et la réduit au silence, pendant de longues années. Jusqu'au jour où croisant son infernal visiteur, elle interroge un passant sur l'identité de celui-ci : «Es el demonio » lui est-il répondu, mais des infirmiers interviennent pour ramener le passant, un aliéné, dans l'espace clos qui lui 
est propre, et les paroles de la sœur ne franchiront sans doute plus l'espace d'une confidence "entre elles ", elle vient de découvrir que du ridicule à la folie il n'a qu'un pas.

L'instance narratrice apparaît ensuite :

- Doctor ¿cree usted en maleficios ? - dije un día a mi amigo el esclarecido profesor Passaman. Gustábame preguntarle, porque de sus respuestas surgía siempre una enseñanza o un relato interesante. (135)

Le dialogue qui suit oppose les croyances superstitieuses de la locutrice aux positions positives de son interlocuteur. La situation est, une fois encore, de soumission : il la tutoie alors qu'elle le vouvoie, elle attend de lui un enseignement, et il va se charger, au moyen d'un récit, de faire disparaître de son esprit "esas absurdas creencias ». Cependant, malgré son autorité, il s'avoue vaincu d'avance :

...Pero, bah! tu las amas, son las golosinas de tu espíritu, y te obstinas en conservarlas. Es inútil.

Oh ! no, querido doctor, refiera usted, por Dios, esa historia ! ¿Quién sabe ? Tal vez me convierta.

- No lo creo. (135)

Et l'on apprend, d'une part, que le champ scientifique est un peu plus tolérant que le champ religieux, puisque son universalité ne repose pas sur une vérité révélée, et absolue, mais sur des procédures d'expérimentation et de vérification théoriquement ouvertes à tous, qui produisent une vérité provisoire. Et d'autre part, que le récit du docteur ne sera pas écouté pour les enseignements qu'il est supposé transmettre, mais pour le plaisir. Enfin, précisons que non seulement il ne suscite pas la conversion espérée, mais qu'il se clôture sur un deuxième aveu d'impuissance, voire d'incompétence :

- ¿Y qué dices de eso ?

- Yo creo en los alfileres de Lorenza.

- Yo creo en la yerba del doctor Boso.

La symétrie des deux répliques finales montre que si la narratrice, après le récit du docteur, n'a pas abandonné ses " absurdes croyances ", lui en revanche s'est vu contraint d'adopter le mode de la croyance dans son rapport aux vérités qu'il donnait auparavant comme positives. L'alternative présente dans le titre « Yerbas y alfileres » est 
répétée à la fin du conte et exhibe au fondement d'une vérité scientifique une croyance commune qui n'est autre que l'expression d'intérêts corporatistes, ou, selon les termes de P. Bourdieu, l'illusio qui définit le champ médical : le Docteur Passaman croit aux herbes de son confrère, le docteur Boso.

De fil en aiguille, ces récits tissent un réseau où sont mis à nus les divers enjeux de la différence sexuelle, et la violence symbolique que son maintien en tant que structure de domination exige. Par ailleurs, la parole qui se déploie est celle, ludique et gratuite, du conteur. Dans «Quien escucha su mal oye » la confession, bien entendu, n'aboutit pas, c'est au contraire le rappel de ce cadre, à la fin du conte, qui est surprenant, voire comique, puisque sept des huit pages du conte sont occupées par le récit en première personne du conspirateur, récit qui, comme le signale Cortázar (1994, 102), accumule les topiques du roman gothique. Et ce rappel ironique creuse brutalement un écart, et revendique l'indécence de la situation : la faute, le châtiment, l'expiation, l'absolution sont autant de prétextes à jouir d'une fable, vidés de tout contenu moral, ils sont explicitement réappropriés par une perspective ludique, littéraire, qui en outre affecte aux tribulations de l'Histoire internationale auxquelles prend part le narrateur-conspirateur une fin intime, domestique et récréative. La faute avouée devient objet de convoitise, la transgression ne produit pas un discours didactique destiné à détourner l'auditoire du péché, au contraire, par le métadiscours qu'engendre le récit cadre elle est privée de toute signification autre que celle, excitante, du fruit défendu.

Le suspens, lui aussi en abyme, a un effet contagieux : l'obscurité qui occulte la scène - comme au théâtre une chute intempestive du rideau, ou dans les romans-feuilletons, l'interruption calculée qui tient le lecteur en haleine - soustrait la scène au regard du narrateur-voyeur et la mise en suspens de son activité attise et multiplie son désir. La tension du désir du personnage-narrateur envahit donc son interlocutrice, qui à son tour la communique à la belle Cristina, et bien évidemment au lecteur qui, lors de l'apparition furtive, à la fin du conte, de cette tierce personne, se trouve placé lui aussi en position de voyeur, témoin indiscret d'une confidence dont il 
n'était pas le destinataire, et spectateur frustré d'une scène dont il ne connaîtra jamais le final. Ainsi, cette fable au titre en forme de proverbe n'enjoint pas à la sagesse mais contraint le lecteur au vice, multipliant en abyme cette position de voyeur dont le désir s'excite d'être indéfiniment différé. Le texte, véritable conspirateur, par son fonctionnement même sème la transgression, il n'est plus "exemplaire" par ce qu'il montre, mais contagieux, diabolique, par la structure spéculaire qu'il exhibe. Alors que l'interdit pèse sur la volonté de savoir depuis le péché originel, elle est ici revendiquée dans cette curiosité contagieuse, et opposée, en tant que point de vue distancié et ludique, a l'illusio à l'origine des relations de possession et de domination - "l'illusio originaire, qui est constitutive de la masculinité, est sans doute à l'origine de la libido dominandi sous toutes les formes spécifiques qu'elle revêt dans les différents champs » (Bourdieu, 1998, 82).

Adviendrait ainsi, dans ces contes, un discours différentiel et non réductible aux orientations hégémoniques qu'il met en scène. La prolifération des récits métadiégétiques crée une sorte de hiatus doxique : la femme, objet du regard, objet du désir et du discours de l'autre, lui est ravie par une parole obscure, inintelligible, issue de son désir à elle. Cette parole autre, objet second - ou objet au deuxième degré - du désir que mettent en scène les contes, conteste à l'institution religieuse et à la science officielle, reconduites ensemble dans l'affabulation, le fantasme et la croyance, le droit de censurer ou limiter son exercice, et s'articule sur une curiosité qui étend son champ d'action de la revendication à l'accès au savoir jusqu'aux dimensions ludico-littéraires, positions distanciées, résolument modernes.

La femme, comme fable masculine exhibée dans ces textes, renvoie l'imaginaire masculin à un ravissement illimité, non sans quelque rire latent. Le culte à la " charla idólatra », comme La cocina ecléctica, la para-littérature fantastique, ou les sciences occultes, seraient quelques aspects du mode mineur (Deleuze et Guatarri, 1975) sur lequel ce discours contradictoire est contraint de dire son devenir. Les normes hégémoniques du discours critique doivent-elles nous conduire à l'ignorance de ce mode, ou devons-nous, pour lire 
ses fables, pratiquer l'anamnèse à laquelle elles nous convient.

\section{BIBLIOGRAPHIE}

BOURDIEU (P.) : Méditations pascaliennes, Paris, Seuil, 1997.

La domination masculine, Paris, Seuil, 1998.

DELeUZE (G.) - GUATTARI (F.) : Kafka. Pour une littérature mineure, Paris, Minuit, 1975

GORRITI (J. M.): Sueños y realidades, 1865 ; Obras completas, IV, Salta, Fundación del Banco del Noroeste, 1993.

HAHN (O.) : El cuento fantástico hispanoamericano en el siglo XIX, México, Premia, 1978 ; 2ème édition : 1982.

LE DEEUFF (M.) : Le sexe du savoir, Paris Aubier, 1998.

ROYO (A.) : (compiladora) Juanamauela, mucho papel, Ed. del Robledal, Salta, 1999.

TERRON DE BELLOMO (H.) : «Literatura fantástica y denuncia social: Juana Manuela Gorriti », Letras femeninas, Vol. XIX- N. 1-2, Primavera-Otoño 1993, Universidad Nebraska-Lincoln.

VERDEVOYE (P.) : «Orígenes y trayectoria de la literatura fantástica en el Río de la Plata », El relato fantástico en España e Hispanoamérica, Enriqueta Morillas Ventura editora, Madrid, Siruela - Quinto Centenario, 1991. 